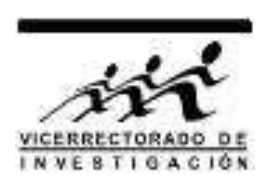

\title{
Cálculo de la magnitud sísmica para la estación de Ñaña
}

\author{
César Jiménez ${ }^{*}$ \\ Fenlab, Facultad de Ciencias Físicas, Universidad Nacional Mayor de San Marcos, Lima, Perú
}

Recibido 03 agosto 2010 - Aceptado 17 octubre 2010

\begin{abstract}
La magnitud sísmica es un parámetro básico que está relacionado con la cantidad de energía liberada por un sismo. La magnitud es función del contenido energético de la señal y de la atenuación por la distancia epicentral. La estación sísmica de Naña (NNA) pertenece a la red internacional Incorporated Research Institutions for Seismology (IRIS), tiene un sensor de banda ancha de 3 componentes. La adquisición de la señal es libre vía internet mediante el protocolo AutoDRM. En este trabajo se desarrolla un algoritmo matemático mediante el método de regresión lineal múltiple. Los datos hipocentrales son obtenidos a partir del catálogo del Instituto Geofísico del Perú (IGP), mientras que las magnitudes se han obtenido del catálogo del National Earthquake Information Center (NEIC). La ecuación de magnitud obtenida está homologada con respecto a la magnitud Mw del NEIC.
\end{abstract}

Palabras claves: sismología, magnitud, Matlab.

\section{Seismic magnitud calculation for Ñaña station}

The seismic magnitude is a basic parameter which is related with the amount of energy released by an earthquake. The magnitude is a function of the signal energy and of the attenuation for the epicentral distance. The seismic station of Naña (NNA) belongs to the international network Incorporated Research Institutions for Seismology (IRIS), has a broadband sensor of three components. The acquisition of the signal is free via internet through AutoDRM protocol. In this work a mathematical algorithm is developed through the multiple linear regression method. The hipocentral data are obtained from the Instituto Geofísico del Perú (IGP) catalog, while the magnitudes are obtained from the National Earthquake Information Center (NEIC) catalog. The magnitude equation obtained is accredit respect to the Mw magnitude of NEIC.

Keywords: seismology, magnitude, Matlab.

\section{Introducción}

La estación sísmica de Naña (NNA) se encuentra localizada a $22 \mathrm{~km}$ al este de la ciudad de Lima. Inició sus operaciones en 1956 como parte de la red mundial de estaciones sísmicas conocida como WWSSN por sus siglas del inglés World-Wide Standard Seismographic Network. En 1989, se inicia el proyecto IDA ((International Deployment of Accelerometers)), que consiste de una red global de banda ancha y digital para sismómetros operados por Cecil H. y Ida M. Green del Institute of Geophysics and Planetary Physics (IGPP), Scripps Institution of Oceanography (SIO) y la University of California, San Diego (UCSD). En 1994 el sistema fue actualizado con un speedometer MK7 y en abril de 2006 por un speedometer MK8. Actualmente, la estación NNA esta certificada como una estación auxiliar

\footnotetext{
*1cesarsud86@hotmail.com
}

\section{del IMS/CTBTO[1].}

La magnitud local se puede usar para cuantificar el tamaño de sismos pequeños y locales, pero para sismos grandes $(M>7.0)$ esta magnitud se satura. En el caso del Instituto Geofísico del Perú (IGP), el servicio sismológico calcula la magnitud (presentada en el Reporte Sísmico Inicial) en base a la duración de la señal sísmica para estaciones de periodo corto. Cuando el evento es muy grande el sistema filtra las componentes frecuenciales de periodo largo (característico de los sismos grandes) y corta la señal del evento antes que éste termine y lo graba en un registro sísmico digital, lo que produce una magnitud subestimada, como se puede apreciar en el cuadro I para los dos últimos grandes terremotos ocurridos en el Perú.

Para cuantificar el tamaño de sismos grandes se de- 
be utilizar la magnitud de momento $M_{w}$ calculada para una estación sísmica de banda ancha triaxial (3 componentes). El momento sísmico relacionado a esta escala de magnitud es proporcional a las dimensiones de la geometría del área de ruptura, proporcionando una mejor idea del tamaño del sismo y la escala de magnitud asociada no se satura

\begin{tabular}{lll}
\hline Evento & $M_{L}$ (IGP) & $M_{w}$ (NEIC) \\
\hline Camaná 2001 & 6.9 & 8.2 \\
Pisco 2007 & 7.0 & 7.9 \\
\hline
\end{tabular}

Tabla 1: Comparación de magnitudes preliminares calculadas por el IGP y NEIC

Para este análisis, se considera que la magnitud de un sismo depende del contenido energético de la señal $E$ para un sismógrafo de banda ancha triaxial y de la atenuación con respecto a la distancia epicentral $D$ y profundidad focal $H$, mediante la ecuación

$$
M=a \log (E)+b \log (D)+c \log (H)+d
$$

donde los parámetros $a, b, c$ y $d$ se obtienen a partir de los datos de varios eventos patrones mediante el método de regresión lineal múltiple.

\section{Energía de una señal}

En muchas aplicaciones, aunque no en todas, las señales están directamente relacionadas con cantidades físicas que capturan potencia y energía de un sistema físico. Por ejemplo, si $v(t)$ e $i(t)$ son, respectivamente, el voltaje y la corriente a través de un resistor $R$, entonces la potencia instantánea es $p(t)=v(t) i(t)$.

La energía total gastada durante un intervalo de tiempo $t_{1}<t<t_{2}$, es

$$
E=\int_{t_{1}}^{t_{2}} p(t) d t
$$

Resulta común y útil usar como convención una terminología similar para potencia y energía para cualquier señal continua $x(t)$ o para cualquier señal discreta $x(n)$. En este caso, la energía total en el intervalo de tiempo $t_{1}<t<t_{2}$ en una señal continua $x(t)$ se define como

$$
E=\frac{1}{2} \int_{t_{1}}^{t_{2}}[x(t)]^{2} d t
$$

De manera similar, la energía total en una señal discreta $x(n)$ en el intervalo de tiempo $1<n<N$ se define como

$$
E=\frac{1}{2} \sum_{n=1}^{N}[x(n)]^{2}
$$

Es importante recordar que el término energía se usa aquí independientemente de si la cantidad de la ecuación anterior está en verdad relacionada con la energía física. No obstante, es conveniente utilizar este término de una manera general. Por lo tanto, la cantidad $E$ representa el contenido energético de la señal sísmica[2].

No es necesario realizar la deconvolución de la señal sísmica para quitarle el efecto del instrumento, puesto que el método de regresión lineal trabaja en forma estadística sobre un conjunto de datos.

\section{Magnitud}

La magnitud es un parámetro relacionado a la energía liberada por el sismo, medida por un sismógrafo. Es un parámetro objetivo que no depende de variables macroscópicas. Existen varios métodos diferentes para medir la magnitud a partir de los sismogramas, cada método funciona en un rango limitado de magnitudes y depende del tipo de sismógrafo. Algunos métodos se basan en las ondas de volumen (que viajan en lo profundo de la estructura de la tierra), unos se basan en las ondas superficiales (que viajan a lo largo de las capas superiores de la tierra), y otros se basan en otras propiedades totalmente diferentes. Sin embargo, todos los métodos están diseñados para concordar en el rango de las magnitudes donde son fiables. Podemos considerar los siguientes tipos de escalas de magnitud, entre otros:

a. Magnitud local $\left(M_{L}\right)$, la primera escala de magnitud sísmica fue desarrollada por Richter en 1930. Richter observó que el logaritmo de la máxima amplitud decae con la distancia. Las observaciones las realizó para el mismo tipo de sismógrafo de torsión Wood-Anderson. El tamaño relativo de los eventos se calcula por comparación a un evento referencial

$$
M_{L}=\log (A)-\log \left(A_{0}\right)
$$

Los eventos mas pequeños que pueden ser registrados tienen magnitudes por encima de cero y los más grandes tienen una magnitud de alrededor de 7 , debido al fenómeno de saturación de la magnitud. La escala de magnitud de Richter sólo debe utilizarse para sismos locales y regionales $(D<600 \mathrm{~km})$ de magnitud menor a 7.0 .

b. Magnitud de ondas de volumen $(m b)$, considera la relación entre la amplitud de las ondas $P$ o $S$ y el periodo principal del registro sísmico para la componente vertical

$$
m b=\log \left(\frac{A}{T}\right)+Q(\Delta, h)
$$

donde $Q$ es el factor de atenuación de la onda sísmica que depende de la distancia epicentral $\Delta$ y de la profundidad $h$. Esta escala es más apropiada para sismos con foco profundo. El rango de magnitud fiable es de 4 a 7 , para sismos mayores la escala se satura. 
c. Magnitud de ondas superficiales $\left(M_{s}\right)$, está basado en la máxima amplitud de las ondas Rayleigh en el rango de frecuencias entre 17 y 22 segundos. Es válido para sismos superficiales $(h<70 \mathrm{~km})$. Esta escala se satura para sismos muy grandes, mayores a 8.0. La ecuación es

$$
M_{s}=\log \left(\frac{A}{T}\right)+1.66 \log (\Delta)+3.3 .
$$

d. Magnitud de Momento $\left(M_{w}\right)$, está relacionada con las dimensiones del área de ruptura a través del momento sísmico $\left(M_{o}\right)$ del terremoto. El momento sísmico se define como

$$
M_{o}=\mu L W U
$$

donde $\mu=4.5 \times 10^{10} \mathrm{~N} / \mathrm{m}^{2}$ es el módulo de rigidez del medio, $L, W$ son las dimensiones de la falla y $U$ es la dislocación de la falla. El momento sísmico puede obtenerse a partir del espectro de la señal sísmica y es proporcional a la amplitud del espectro para bajas frecuencias en la escala logarítmica. La magnitud de momento está dado por

$$
M_{w}=\frac{\log \left(M_{o}\right)}{1.5}-10.73
$$

Esta escala, desarrollada por Kanamori, no se saturará debido a que $M_{o}$ tampoco se satura. Para sismos grandes es mejor utilizar esta escala, independientemente de la distancia epicentral[3].

e. Magnitud energía $\left(M_{e}\right.$, está basada en la cantidad de energía símica emitida o radiada por el terremoto. Esta escala no se satura y se puede emplear para cuantificar sismos mayores a 3.5, independientemente de la distancia epicentral. A partir del sismograma puede obtenerse el contenido energético de la señal sísmica, debe considerarse todo el paquete de ondas, tanto las ondas de volumen como las superficiales. Luego se aplica la ecuación (1).

\section{Método de regresión lineal múltiple}

Muchos modelos no-lineales pueden aproximarse mediante modelos lineales bajo ciertas condiciones. Ciertamente, el procedimiento más objetivo para ajustar una recta a un conjunto de datos presentados en un diagrama de dispersión se conoce como el método de los mínimos cuadrados. La recta resultante presenta dos características importantes:

a. La suma de las desviaciones verticales de los $n$ puntos a partir de la recta de ajuste es nula.

$$
\sum_{i=1}^{n}\left(f-f_{i}\right)=0
$$

b. La suma de los cuadrados de dichas desviaciones es mínima. Ninguna otra recta daría una suma menor de las desviaciones elevadas al cuadrado. Luego, el procedimiento consiste en minimizar la suma de los cuadrados.

Sea la función $f=f(x, y, z)$ de 3 variables independientes dada por

$$
f=a x+b y+c z+d
$$

donde $a, b, c$ y $d$ son parámetros a determinar. Sea $S$ la suma de los cuadrados de las diferencias

$$
\begin{gathered}
S=\sum_{i=1}^{n}\left(f-f_{i}\right)^{2} \\
S=\sum_{i=1}^{n}\left[\left(a x_{i}+b y_{i}+c z_{i}+d\right)-f_{i}\right]^{2} .
\end{gathered}
$$

Minimizando la sumatoria $S$ tenemos

$$
\begin{aligned}
& \frac{\partial S}{\partial a}=2 \sum_{i=1}^{n}\left[\left(a x_{i}+b y_{i}+c z_{i}+d\right)-f_{i}\right] x_{i}=0 \\
& \frac{\partial S}{\partial b}=2 \sum_{i=1}^{n}\left[\left(a x_{i}+b y_{i}+c z_{i}+d\right)-f_{i}\right] y_{i}=0 \\
& \frac{\partial S}{\partial c}=2 \sum_{i=1}^{n}\left[\left(a x_{i}+b y_{i}+c z_{i}+d\right)-f_{i}\right] z_{i}=0 \\
& \frac{\partial S}{\partial d}=2 \sum_{i=1}^{n}\left[\left(a x_{i}+b y_{i}+c z_{i}+d\right)-f_{i}\right]=0 .
\end{aligned}
$$

Luego, ordenando y dando forma, obtenemos el siguiente sistema de cuatro ecuaciones lineales para calcular los parámetros $a, b, c$ y $d$

$$
\begin{gathered}
a \sum_{i=1}^{n} x_{i}^{2}+b \sum_{i=1}^{n} x_{i} y_{i}+c \sum_{i=1}^{n} x_{i} z_{i}+d \sum_{i=1}^{n} x_{i}=\sum_{i=1}^{n} x_{i} f_{i} \\
a \sum_{i=1}^{n} x_{i} y_{i}+b \sum_{i=1}^{n} y_{i}^{2}+c \sum_{i=1}^{n} y_{i} z_{i}+d \sum_{i=1}^{n} y_{i}=\sum_{i=1}^{n} y_{i} f_{i} \\
a \sum_{i=1}^{n} x_{i} z_{i}+b \sum_{i=1}^{n} y_{i} z_{i}+c \sum_{i=1}^{n} z_{i}^{2}+d \sum_{i=1}^{n} z_{i}=\sum_{i=1}^{n} z_{i} f_{i} \\
a \sum_{i=1}^{n} x_{i}+b \sum_{i=1}^{n} y_{i}+c \sum_{i=1}^{n} z_{i}+d n=\sum_{i=1}^{n} f_{i}
\end{gathered}
$$

donde las variables $x, y, z$ están relacionadas mediante las ecuaciones

$$
\begin{aligned}
& x=\log (E) \\
& y=\log (D) \\
& z=\log (H)
\end{aligned}
$$

Luego de procesar los datos y resolver el sistema de cuatro ecuaciones lineales se obtienen los parámetros $a$, $b, c$ y $d$. De esta forma obtenemos una ecuación para el cálculo de la magnitud homologada con respecto a la magnitud de momento $M_{w}$. 


\section{Adquisición de la señal}

Las señales sísmicas de la red IRIS están disponibles en internet y la adquisición se realiza mediante el protocolo de comunicación AutoDRM. El procedimiento de adquisición se explica a continuación[4]:

I. Ingresar a una cuenta de correo electrónico institucional que no sea de Hotmail ni de Yahoo.

II. Abrir y editar un nuevo mensaje cuyo contenido es el siguiente:

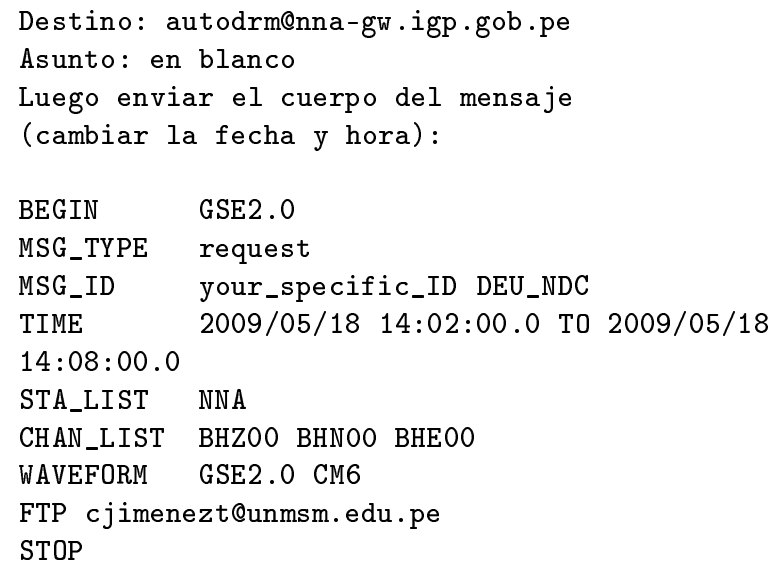

III. Aproximadamente después de 30s se tendrá la respuesta, vía correo electrónico.

IV. Transferir vía FTP del servidor a su PC:

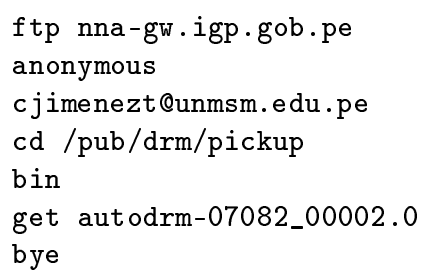

IV. Conversión de formato: abrir el programa Matlab, ubicarse en el directorio correspondiente.

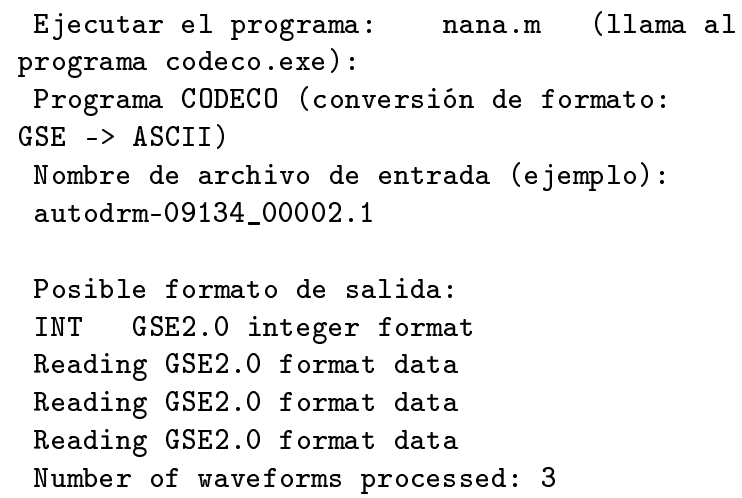

V. Finalmente, analizar la señal y calcular la magnitud.
También es posible obtener las formas de onda de sismos históricos a partir de los catálogos del NEIC o IRIS, empleando el software Jweed V3.0, el cual trabaja en plataforma Java[5].

\section{Datos}

Se han seleccionado 27 eventos sísmicos ocurridos entre el año 2008 y 2010 en el territorio nacional y algunos fuera del Perú. Los datos han sido pre-procesados, es decir se ha calculado la energía de la señal $E$, la distancia entre el epicentro y la estación $D$ y la profundidad focal $H$ calculada por el IGP[6]. La magnitud corresponde a los datos calculados por el NEIC[7]. Tal como se muestra en el cuadro II (donde $D$ y $H$ están en $\mathrm{km})$ :

\begin{tabular}{rrrrrr}
\hline Fecha & GMT & $\log (\mathrm{E})$ & $\log (\mathrm{D})$ & $\mathrm{H}$ & $\mathrm{Mw}$ \\
\hline 07 Feb 07 & $18: 55$ & 10.2685 & 2.9469 & 23 & 5.5 \\
01 Nov 08 & $20: 40$ & 13.3093 & 1.7334 & 52 & 4.5 \\
19 Ene 09 & $00: 02$ & 12.1911 & 2.2788 & 37 & 4.8 \\
02 Feb 09 & $17: 53$ & 14.1571 & 2.2519 & 35 & 6.0 \\
15 Feb 09 & $10: 04$ & 12.7054 & 2.9101 & 33 & 6.1 \\
26 Mar 09 & $17: 35$ & 12.5616 & 2.9320 & 34 & 5.8 \\
17 Abr 09 & $02: 08$ & 11.8935 & 3.0314 & 17 & 6.1 \\
18 May 09 & $14: 01$ & 12.6601 & 2.6599 & 17 & 5.8 \\
26 May 09 & $06: 38$ & 12.2388 & 2.4472 & 39 & 5.4 \\
28 May 09 & $08: 24$ & 13.8968 & 3.5218 & 10 & 7.3 \\
02 Jun 09 & $21: 37$ & 10.9063 & 2.6294 & 101 & 4.9 \\
06 Jun 09 & $21: 51$ & 10.9389 & 2.6031 & 136 & 5.2 \\
13 Jun 09 & $20: 10$ & 10.5411 & 2.8960 & 33 & 5.2 \\
15 Jun 09 & $13: 04$ & 13.3151 & 2.3010 & 51 & 5.4 \\
01 Ago 09 & $23: 07$ & 13.0374 & 2.1761 & 113 & 5.2 \\
03 Ago 09 & $20: 10$ & 12.3538 & 2.4654 & 55 & 5.3 \\
27 Ago 09 & $14: 56$ & 10.8504 & 2.9370 & 37 & 5.3 \\
13 Nov 09 & $07: 27$ & 11.0042 & 3.1813 & 608 & 5.8 \\
13 Nov 09 & $03: 05$ & 12.9338 & 3.0573 & 25 & 6.5 \\
24 Dic 09 & $14: 01$ & 12.5856 & 2.7067 & 82 & 5.6 \\
03 Ene 10 & $20: 39$ & 13.8244 & 2.5502 & 104 & 5.7 \\
05 Ene 10 & $01: 34$ & 10.6398 & 2.7657 & 50 & 5.0 \\
25 Ene 10 & $22: 52$ & 13.3638 & 2.6532 & 154 & 5.9 \\
28 Ene 10 & $08: 04$ & 10.2671 & 3.2185 & 106 & 5.8 \\
27 Feb 10 & $06: 34$ & 16.8579 & 3.4464 & 35 & 8.8 \\
09 Oct 10 & $14: 04$ & 10.2811 & 3.0086 & 121 & 5.3 \\
17 Oct 10 & $08: 27$ & 10.8518 & 2.6803 & 93 & 5.2 \\
\hline
\end{tabular}

Tabla 2: Datos sísmicos pre-procesados.

En la Fig. 1 se muestra la señal sísmica correspondiente al sismo ocurrido al norte de Iquique en Chile el 13 Nov 2009, con una magnitud de 6.5 según el Neic y 6.4 según este trabajo. 


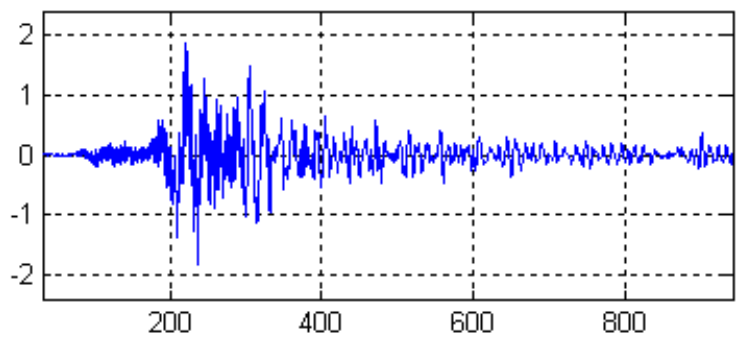

Figura 1: Señal del sismo de Chile 13 de noviembre del 2009, cuentas vs. tiempo (s).

Los datos del cuadro II pueden grabarse en el archivo de texto datos.txt. Luego, el siguiente programa en Matlab realiza la resolución del sistema de ecuaciones (12), (13), (14) y (15) y calcula los parámetros $a, b, c$ y $d$ :

\section{$\%$ Programa magnit2.m}

$\% M=a * \log (E)+b * \log (D)+c * \log (H)+d$

load datos.txt;

$\mathrm{x}=\operatorname{datos}(:, 1)$;

$\mathrm{y}=\operatorname{datos}(:, 2)$;

$z=\log 10(\operatorname{datos}(:, 3))$;

$f=\operatorname{datos}(:, 4)$;

$\mathrm{n}=$ length $(\mathrm{x})$;

$\mathrm{A}=[\operatorname{sum}(\mathrm{x} \cdot * \mathrm{x}) \operatorname{sum}(\mathrm{x} \cdot * \mathrm{y}) \operatorname{sum}(\mathrm{x} \cdot * \mathrm{z})$

$\operatorname{sum}(x)$;

$\operatorname{sum}(x \cdot * y) \operatorname{sum}(y \cdot * y) \operatorname{sum}(y \cdot * z) \operatorname{sum}(y) ;$

$\operatorname{sum}(x \cdot * z) \operatorname{sum}(y \cdot * z) \operatorname{sum}(z \cdot * z) \operatorname{sum}(z) ;$

$\operatorname{sum}(x) \quad \operatorname{sum}(y) \quad \operatorname{sum}(z) \quad n \quad] ;$

$B=[\operatorname{sum}(x \cdot * f) \operatorname{sum}(y \cdot * f) \operatorname{sum}(z \cdot * f)$ $\operatorname{sum}(f)]^{\prime} ;$

$\mathrm{X}=\operatorname{inv}(\mathrm{A}) * \mathrm{~B}$;

$\mathrm{a}=\mathrm{X}(1)$

$\mathrm{b}=\mathrm{X}(2)$

$c=X(3)$

$\mathrm{d}=\mathrm{X}(4)$

\section{Resultados}

Luego de procesar los datos mediante los algoritmos y programas correspondientes desarrollados en el entorno de programación Matlab[8], se obtienen los valores de los parámetros, correspondientes a la ecuación de la magnitud sísmica, Ec.(1):

$$
\begin{aligned}
a & =0.3863 \\
b & =1.5160 \\
c & =-0.0397 \\
d & =-3.1323
\end{aligned}
$$

Las condiciones de aplicabilidad de los coeficientes calculados son: magnitud menor a 4.5 , distancia epicentral menor a $30^{\circ}$, no hay restricción con respecto a la profundidad focal. La desviación estándar para la diferencia entre los valores de magnitud observados y calculados es

$$
\sigma=0.1675 \text {. }
$$

En el cuadro 3, se muestran algunos resultados comparativos de las magnitudes de eventos sísmicos ocurridos y correspondientes a los años 2009 y 2010,

\begin{tabular}{lrrl}
\hline Fecha & NEIC & P.T. & Lugar \\
\hline 02 Feb 09 & 6.0 & 5.8 & Pisco \\
15 Feb 09 & 6.0 & 6.1 & Sechura \\
26 Mar 09 & 5.8 & 6.0 & Paita \\
17 Abr 09 & 6.1 & 6.0 & Tacna \\
18 May 09 & 5.8 & 5.7 & Ica \\
26 May 09 & 5.1 & 5.2 & Ica \\
28 May 09 & 7.3 & 7.4 & Honduras \\
02 Jun 09 & 4.8 & 5.0 & Puquio \\
13 Jun 09 & 5.4 & 5.3 & Mollendo \\
15 Jun 09 & 5.4 & 5.4 & Pisco \\
01 Ago 09 & 5.1 & 5.1 & Lima II \\
03 Ago 09 & 5.3 & 5.3 & Ica \\
27 Ago 09 & 5.2 & 5.4 & Sechura \\
13 Nov 09 & 5.8 & 5.7 & Bolivia \\
13 Nov 09 & 6.5 & 6.4 & Chile \\
24 Dic 09 & 5.6 & 5.7 & Chala \\
03 Ene 10 & 5.7 & 5.9 & Huaraz \\
05 Ene 10 & 5.0 & 5.1 & Chala \\
25 Ene 10 & 5.8 & 5.8 & Pucallpa \\
28 Ene 10 & 5.8 & 5.6 & Argentina \\
27 Feb 10 & 8.8 & 8.5 & Chile \\
09 Oct 10 & 5.3 & 5.3 & Peru-Ecuador \\
\hline
\end{tabular}

Tabla 3: Magnitudes comparativas entre nuestros cálculos y del NEIC

Es notoria la buena correlación entre los valores de la magnitud para el terremoto de Honduras del 28 de mayo de 2009, para el cual el NEIC calcula una magnitud de $7.3 \mathrm{Mw}$ y nuestro cálculo 7.4. Para otros eventos se tiene una diferencia de 0.2 , lo cual está casi dentro de los márgenes de error calculados mediante la desviación estándar. La rutina en Matlab está escrito como

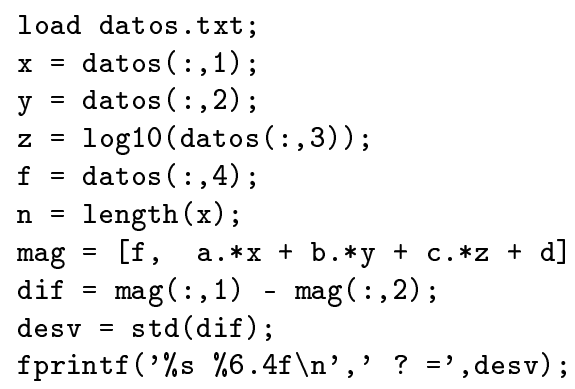


El 12 de enero de 2010 la nación de Haití fue severamente golpeada por un terremoto. La magnitud preliminar del NEIC fue de 7.3 Mw. El Centro Sismológico Europeo Mediterráneo calculó una magnitud de 7.1 Mw, mientras que nuestro cálculo fue 7.4.

Si el sismo es muy grande y su epicentro está cerca de la estación, es probable que la señal sísmica se sature. Tal es el caso del terremoto de Pisco del 15 de agosto de 2007, como se observa en la Fig. 2,

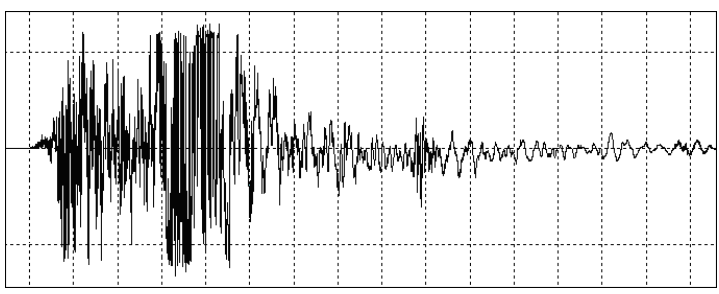

Figura 2: Señal del sismo de Pisco 2007.

En este caso, se debe tomar la señal de una estación sísmica con mayor distancia epicentral $D$. En caso contrario, se tendrá una magnitud subestimada.

\section{Conclusiones}

En este trabajo se presenta un método para realizar el cálculo de la magnitud en forma rápida luego de producirse un sismo a nivel nacional (o internacional). La desviación estándar es $\sigma=0.16$

La magnitud calculada depende del contenido energético de la señal sísmica, pero está homologada con respecto a la magnitud momento (Mw) del NEIC. La ventaja de la escala $\mathrm{Mw}$ es que no se satura.

No es necesario realizar la deconvolución de la señal para quitarle el efecto del instrumento. La formulación se puede aplicar para cualquier estación, para lo cual, se debe remover la respuesta del instrumento.

Se considera que la magnitud tiene una relación lineal con el logaritmo decimal de: la energía de la señal, la distancia epicentral y la profundidad focal.

El NEIC calcula una buena magnitud (para Mw $>5.0$ ), pero no calcula un buen epicentro (para sismos en el Perú). Mientras que el IGP calcula un buen epicentro, pero no calcula una buena magnitud para sismos mayores a 5.0.

El Catálogo Sísmico del IGP debe ser revisado para recalcular las magnitudes mayores a 5.0 y homologarlas con respecto a la magnitud Mw. El cálculo de la magnitud del IGP está basado en la duración de la señal para estaciones de periodo corto, y esto se satura para sismos grandes.

Los parámetros de la ecuación obtenidos son únicos para la estación sísmica de Ñaña o para otra estación de similar función de transferencia del sistema. Si el

equipo se cambia, entonces se tendrá que calcular los nuevos parámetros de la ecuación.

La ecuación obtenida es factible de ser mejorada con la inclusión de nuevos datos para sismos recientes (posteriores a octubre de 2010).

\section{Apéndice}

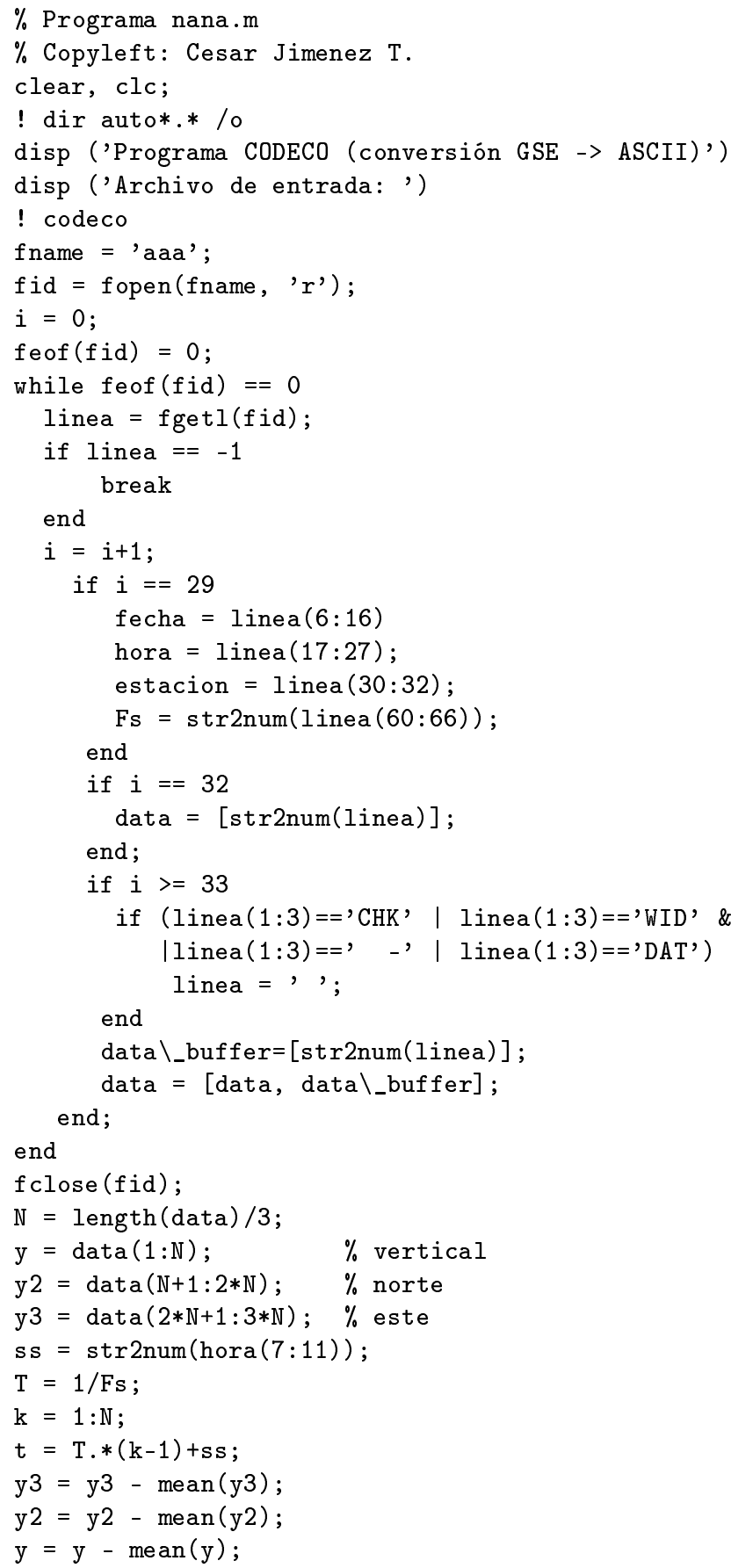



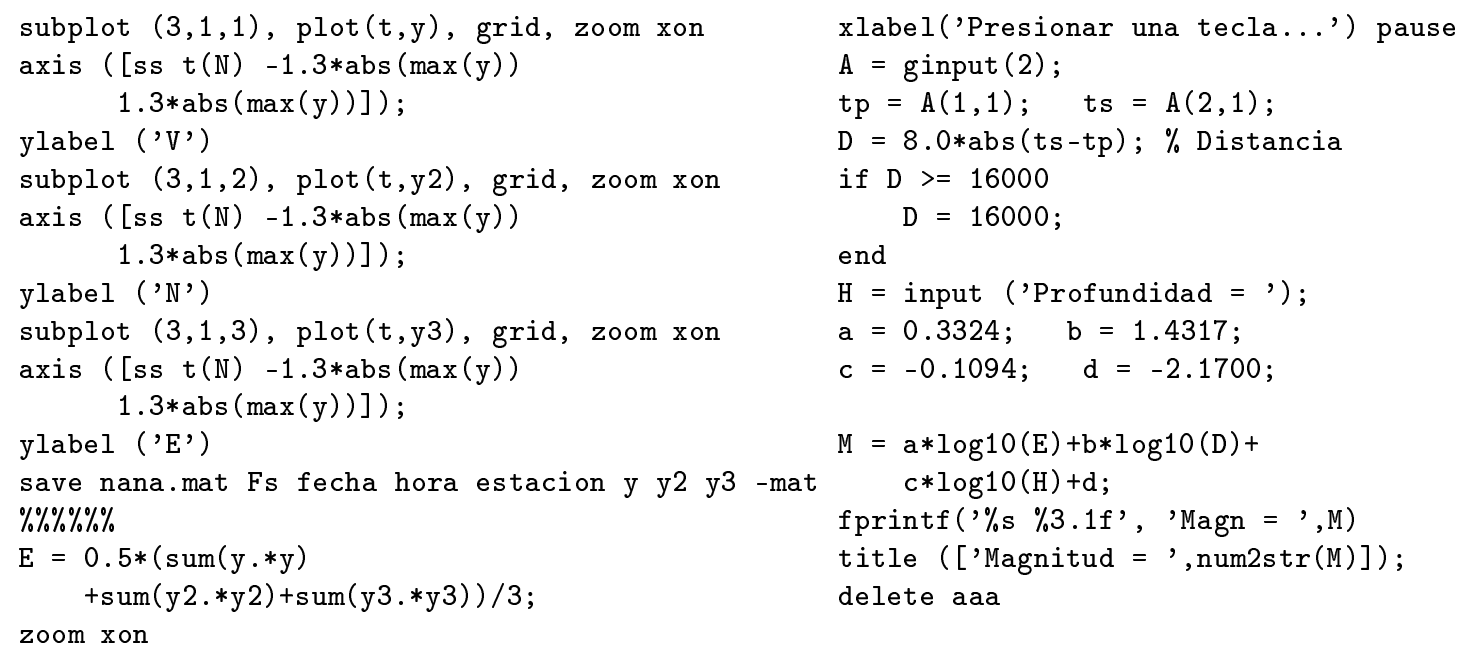

\section{Referencias}

[1] Edmundo Norabuena, A brief history of the seismographic station at Ñañ, Peru; IRIS Newsletter 13, 8 (1994). También en http://ida.ucsd.edu/Stations/index.html.

[2] Alan V. Oppenheim y Alan S. Willsky; Señales y Sistemas, Ed. Prentice Hall, México (1998).

[3] Thorne Lay y Terry C. Wallace; Modern Global Seismology, Academic Press Inc., California (1995).

[4] AutoDRM User Guide: http://www.seismo.ethz . edu/autodrm/autodrm_help.html
[5] C. Laughbon, Jweed Software, IRIS-DMC http://www.iris.edu/manuals/jweed/

[6] Página web del Servicio Sismológico (IGP): http://khatati.igp.gob.pe/Webs/cns06/sismo/ IGPSIS/sismo.htm

[7] NEIC, USGS. Catálogo de terremotos en línea: http://neic.usgs.gov/neis/epic/epic_rect.html

[8] C. Jiménez, Procesamiento digital de señales sísmicas con Matlab; Rev. Inv. Fis. 10, 45 (2007). 